\title{
VITAL CELEBRATES FIRST DECADE IN STYLE
}


NEWS 